\title{
Research on the Formation and Promotion Mechanism of Science and Technology Intermediary Service System
}

\author{
Xiansheng Liu $^{1, \text { a }}$ \\ ${ }^{1}$ Xi'an International University, Xi'an, Shaanxi, 710077 \\ ${ }^{a}$ email
}

Keywords: Intermediary Service System, Promotion Mechanism, Formation Technology

\begin{abstract}
Based on a brief overview of the technology intermediary service industry and its constituent system, this paper expounds the formation process of Chinese science and technology intermediary service system, and analyzes the factors that hinder the development of science and technology intermediary service industry. And discuss the factors that hinder its development and its cause to find out its solutions, to make suggestions on how to improve the structure mechanism of Chinese science and technology intermediary service system and promote the development of Chinese science and technology intermediary service industry.
\end{abstract}

\section{Introduction}

The improvement of enterprise's scientific and technological innovation ability is closely related to the composition of enterprise science and technology intermediary service system. The party's 17th Congress made it clear that the development of enterprises is inseparable from the innovation of science and technology and enterprise innovation, must be applied to the scientific and technological achievements in the modernization of enterprises to the development of enterprises as the main body, focusing on market-oriented role, improve the level of productivity of enterprise development, combining innovation and practice to promote the application of innovative elements in enterprise development. At the same time, the development of science and technology intermediary services, to promote Chinese agriculture, industry and tertiary industry development, promote Chinese modernization process, improve our scientific and technological innovation and national strength, enhance the overall quality of national industries and increase national output, optimize the national industry machine structure and other aspects are of great significance.

Technology intermediary organizations are also known as technology intermediaries. There are many definitions of science and technology intermediary organizations, such as Ma Songyao (2004) that science and technology intermediary organizations refers to the main body of scientific and technological innovation - enterprises and universities, research institutions and other social subjects to provide social and professional services to support scientific and technological innovation activities and to promote the industrialization of scientific and technological achievements of the institutions. Other scholars from different angles put forward the definition of science and technology intermediary organizations, such as science and technology intermediary organizations is from the scientific and technological achievements to the industrialization of the realization of science and technology and economic integration to provide the necessary information, resources, services, small and medium enterprises, Science and technology small and medium enterprises as the object of non-governmental organizations. We believe that science and technology intermediary organizations refer to the main body of scientific and technological innovation to provide social, professional services to support and promote innovation activities of the body. This kind of organization mainly carries out the information exchange, decision-making consultation, resource allocation, technical service and technology visa directly related to the scientific and technological innovation, and plays a key role in the knowledge flow and technology transfer between the government and all kinds of innovation subjects and markets. Promote the role, can effectively reduce the cost of innovation, resolve the risk of innovation, accelerate the transformation of scientific and technological achievements and improve the overall scientific and 
technological innovation.

\section{Development Status and Problems of Science and Technology Intermediary Service Industry in China}

After 30 years of reform and opening up the modernization of construction, science and technology intermediary services in China has been significant development. With the continuous progress of the modernization process, the continuous improvement of the market economy system, the continuous expansion of enterprise scale, science and technology intermediary service organizations have also expanded the scale. With the continuous improvement of science and technology intermediary service system, the main body and function of science and technology intermediary service has also formed a variety of characteristics, the emergence of a lot of high-tech talent in science and technology intermediary, the industry's overall management level has also been significantly improved. Although in many ways have made significant progress, but there are still many drawbacks, such as industry management mechanism, market mechanism, organizational system and so on imperfect, policies and measures can not be effectively implemented, the overall quality of science and technology intermediary team is not higher. To promote the sustainable development of Chinese science and technology intermediary service industry, we must make this problem be improved and resolved, the formation of a good science and technology intermediary service system, to develop a correct and reasonable policy program, science and technology intermediary services and promotion mechanism, so that the overall development of intermediary services.

With the continuous trend of economic globalization, the continuous progress of the process of world economic integration, the rapid development of domestic market economy, and the emergence of new problems represented by environmental issues, science and technology intermediary service industry has returned to the public view and become many countries and regions concerned about the hot issues. The establishment of a sound science and technology intermediary service system is of vital importance to the development of Chinese science and technology intermediary service industry. The establishment of this system is based on the service organization of science and technology intermediary, through the policy measures, strategic decision-making, organizational resources and other aspects of the unified system, has important practical significance, but its development is still subject to quality and quantity of the two. The limitations of the prevailing constraints. In terms of quantity, the production, trading volume and consumption expansion of science and technology intermediary service organizations are outstanding, and the performance is difficult for financing, the cost of production and transaction is high and the price comparability is poor. These problems have seriously restricted the development of science and technology intermediary organizations. Open up and scale up. In terms of quality, the quality of products and services provided by science and technology intermediary organizations is uneven, the overall level needs to be improved, and the quality standards and certification system, quality assurance and technical service system are not perfect, highlighting the development of science and technology intermediary organizations The market awareness, technology lag phenomenon, which also affects its further occupation and expansion of the market. It is clear that the number and quality of technology intermediaries are related to each other. Most of the products provided by the technology intermediary service industry are high-tech, one-time investment is large, there is a large development and market risk, which restricts the technological progress of these products; in turn, due to technology intermediaries products and services Will affect its economic use costs and consumer confidence, hinder the formation of the scale of the market, to further improve its production and transaction costs, increase the development risk and reduce the interest of investors and developers, causing market failure. It resulted in the development of science and technology intermediary service industry in the vicious cycle. 


\section{Measures to Solve the Problems Arising from the Formation of the Intermediary Service System}

To achieve the development of science and technology intermediary service industry, we must rely on strong policy support, through the support of the government, there is no worries about the development of Chinese science and technology intermediary services, mining Chinese science and technology intermediary services industry development potential, under the influence of independent growth of science and technology intermediary services, guide and promote science and technology intermediary service industry innovation. Therefore, many countries will develop appropriate measures to control the development of science and technology intermediary services from all levels of policy intervention, through the government's macro-control to promote the mechanism of research and technology intermediary service system to form a combination of purpose, so as to protect Chinese intermediary services the rapid development of the system.

Analysis on the Mechanism of Promoting the Development of Science and Technology Intermediary Service System. The perfect organizational structure is the organization and protection of the formation and development of science and technology intermediary service system. We must pay attention to the external mechanism of science and technology intermediary services, adhere to the hematopoietic mechanism, by promoting and guiding the organic combination of different mechanisms to promote the healthy development of science and technology intermediary industry.

Pay Attention to the external environment construction technology intermediary service economy externalities are very obvious. In general, the traditional conventional technology research and development due to serious dislocation with the market, making the conversion rate of scientific and technological achievements is low, so show more negative externalities. The technology intermediary service has constructed the communication and docking platform in the aspects of research and development and the practical application of scientific and technological achievements, which reduces the market transaction cost and improves the overall allocation efficiency of social resources, thus showing obvious positive externalities. But in reality the external cost of technology research and development is not included in the final accounting costs of its products and services, as well as the performance evaluation of these R \& D institutions; the contrary, science and technology intermediary services in the production of social environmental benefits at the same time, the initial cost of the independent technology intermediary service organizations to bear. Therefore, in the market competition, the strong contrast makes science and technology intermediary service organizations tend to be disadvantaged, affecting the optimal allocation of social science and technology resources. Obviously, the government's policy on science and technology intermediary should be based on the public interest as the core, through a reasonable tax collection and compensation policy, the cost of science and technology intermediary services to all scientific and technological research and development of products and services to achieve scientific and technological research and development product and service costs of the rational calculation of science and technology intermediaries and conventional technology research and development to create a fair competitive environment, and promote scientific and technological structure optimization and technological innovation.

Pay attention to the external environment construction technology intermediary service economy externalities are very obvious. In general, the traditional conventional technology research and development due to serious dislocation with the market, making the conversion rate of scientific and technological achievements is low, so show more negative externalities. The technology intermediary service has constructed the communication and docking platform in the aspects of research and development and the practical application of scientific and technological achievements, which reduces the market transaction cost and improves the overall allocation efficiency of social resources, thus showing obvious positive externalities. But in reality the external cost of technology research and development is not included in the final accounting costs of its products and services, as well as the performance evaluation of these R \& D institutions; the contrary, science and 
technology intermediary services in the production of social environmental benefits at the same time, The initial cost of the independent technology intermediary service organizations to bear. Therefore, in the market competition, the strong contrast makes science and technology intermediary service organizations tend to be disadvantaged, affecting the optimal allocation of social science and technology resources. Obviously, the government's policy on science and technology intermediary should be based on the public interest as the core, through a reasonable tax collection and compensation policy, the cost of science and technology intermediary services to all scientific and technological research and development of products and services to achieve scientific and technological research and development product and service costs of the rational calculation of science and technology intermediaries and conventional technology research and development to create a fair competitive environment, and promote scientific and technological structure optimization and technological innovation.

\section{Conclusion}

Pay attention to the external environment construction technology intermediary service economy externalities are very obvious. In general, the traditional conventional technology research and development due to serious dislocation with the market, making the conversion rate of scientific and technological achievements is low, so show more negative externalities. The technology intermediary service has constructed the communication and docking platform in the aspects of research and development and the practical application of scientific and technological achievements, which reduces the market transaction cost and improves the overall allocation efficiency of social resources, thus showing obvious positive externalities. But in reality the external cost of technology research and development is not included in the final accounting costs of its products and services, as well as the performance evaluation of these $\mathrm{R} \& \mathrm{D}$ institutions; the contrary, science and technology intermediary services in the production of social environmental benefits at the same time, the initial cost of the independent technology intermediary service organizations to bear. Therefore, in the market competition, the strong contrast makes science and technology intermediary service organizations tend to be disadvantaged, affecting the optimal allocation of social science and technology resources.

\section{Acknowledgements}

Fund: 2017 Shaanxi Science and Technology Association High-End Think Tank Project, build science and technology service system to promote social innovation and entrepreneurship

\section{References}

[1] Luo Guanglin, Yu Xiangping, Lu Jiangdong. Status Quo and Development of Science and Technology Intermediary Service System in Zhejiang Province[J]. Journal of Scientific Research Management 2008 (03)

[2] Li Xin, Zou Lifui.Study on the Motive Mechanism of the Development of Science and Technology Intermediary Service System [J]. Science and Technology Progress and Countermeasures. 2008 (04)

[3] Guo Peng, Cheng Fei. Status Quo of Science and Technology Intermediary Organizations and Its Development Countermeasures[J]. Technology and Innovation Management. 2006 (04)

[4] Zhang Xiaofen, Zhang Daoying.Study on the Development of Liaoning Science and Technology Intermediary Service System [J]. Journal of Liaoning Institute of Technology. 2006 (02)

[5] Gao Yuyu, Chen Yugang, Wang Jianrong, Xu Jianchun. Development and research of science and technology intermediary service[J]. Reform and Strategy. 2005 (10)

[6] Zhao Min. Sichuan science and technology intermediary organizations development countermeasures and suggestions [J]. Decision Advisory Newsletter. 2005 (02) 
[7] Luo Guanglin. Zhejiang Province science and technology intermediary service system construction research [J]. Zhejiang Journal. 2005 (02) 